\title{
La reconstrucción de un pensamiento \\ y una praxis crítica en la psicología
}

latinoamericana

Ignacio Dobles Oropeza

Universidad de Costa Rica

Colectivo Costarricense de Psicología de la Liberación

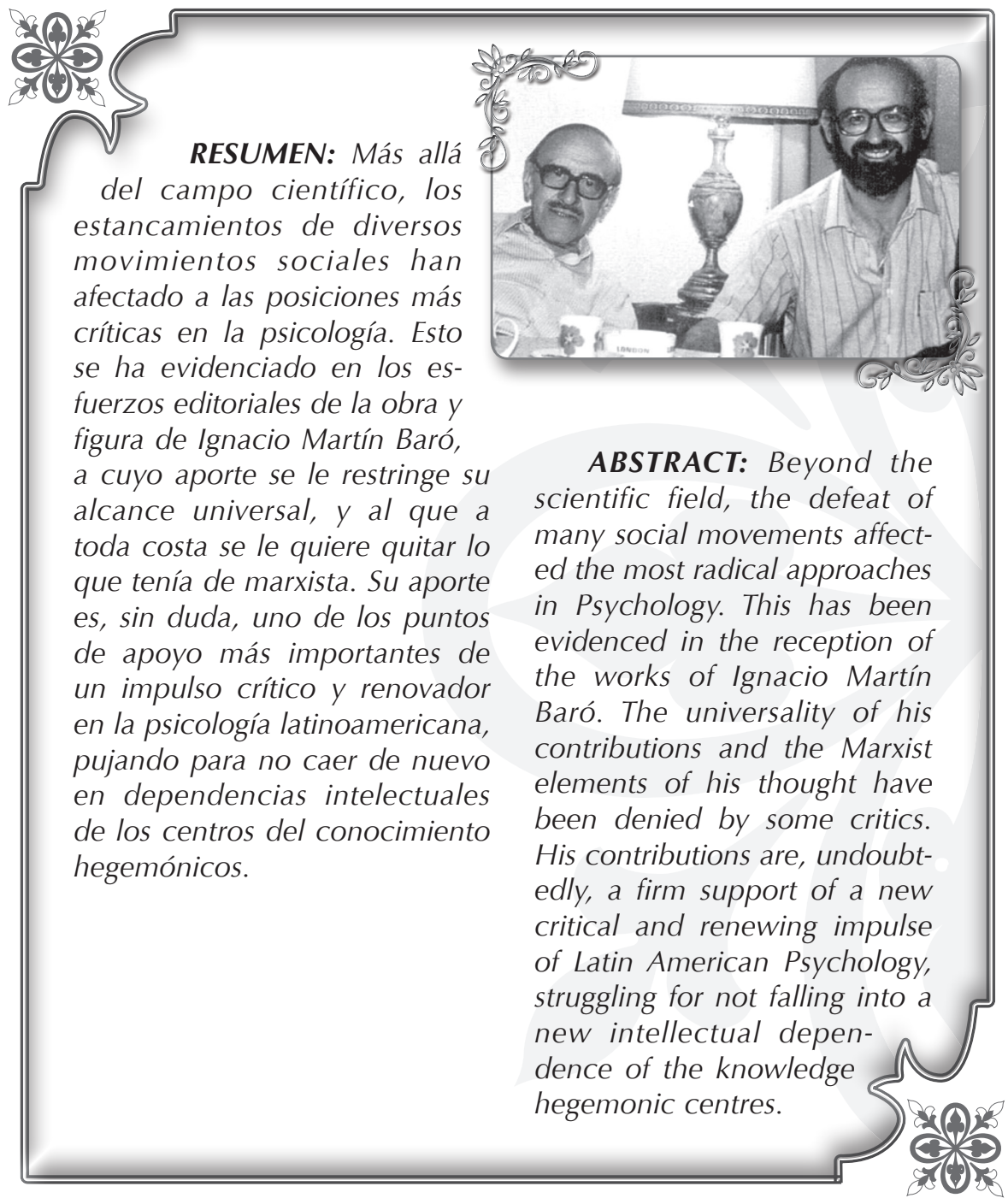


C omo se ha señalado tantas veces, el discurso psicológico, con su individualismo metodológico, y con sus supuestos hedonistas acerca de la "naturaleza humana", a la par de una visión homeostática de la realidad social, ha sido extremadamente funcional, en términos generales, para el orden social imperante, y suele aplicarse, como ha escrito Ibáñez (2001), como dispositivo autoritario y normativo. Sirve, por ejemplo, con toda claridad, para achacar los problemas de la estructura social al individuo o a la familia (Leandro y Montoya, 2006).

Ha sido sustento ideológico eficaz, plato fuerte, para la perspectiva neoliberal, al enfatizar la supuesta libertad del individuo en el orden (desorden) imperante, y al avalar y naturalizar aquello de que la mano invisible del mercado se encarga de traer el bienestar de todos, si tan solo cada quien se dedica a promover su propio bienestar personal.

Asumiendo, muchas veces de manera implícita, esta concepción acerca de la "naturaleza humana" le termina haciendo un enorme servicio a un orden social deshumanizado. El problema, claro está, es que a actual crisis del sistema (mercados) y su abordaje por parte de las instancias de poder global, en que hasta el gobierno de Bush interviene estatalmente los bancos, parece, desde esta óptica reificada, hasta contrafáctico. Pero estos hechos de la macro crisis no quieren decir que la perspectiva neoliberal de la vida no esté fuertemente instalada en cotidianidades e imaginarios. Ya Marx, en su tiempo, se refería a los mercados como la "religión cotidiana".

Habría que enfatizar este punto: hay supuestos psicológicos que asientan, con eficacia, en los imaginarios y las cotidianidades, del discurso neoliberal. No en vano podemos encontrar una veta muy fuerte de psicologismo, que apunta en esta dirección, en los economistas neoliberales.

En la historia de la psicología como disciplina han surgido, en diferentes coyunturas, intentos importantes de críticas y redefiniciones de esa funcionalidad adaptativa de una disciplina en la que el cambio es asunto cardinal, pero individual, inmovilizando, gatopardescamente, al conjunto social como un todo. No se trata, como lo denunciaban algunos autores a principios de los setenta, de la irrelevancia de la psicología, sino de a quien ha servido y para qué.

Ha cumplido funciones valiosas para el capitalismo, en los diferentes establecimientos en que opera lo que se ha llamado el complejo Psi (en el sentido de todas las instituciones, legitimaciones, instituciones formativas, profesionales, y diversos ámbitos en que se expresan discursos y prácticas de los psicólogos y psicólogas), cuando, como discutió en su momento Kenneth Gergen (1996), se ha combinado una teoría armónicamente con una metateoría, 
y con un instrumental metodológico, como hizo el conductismo de principios y mediados del siglo veinte.

Tenemos, por otro lado, y en signo contrario, una larga historia en el psicoanálisis, de intentar desplazarse mas allá de la cuidadosa realpolitik instalada originariamente por Freud como metodología de protección de una institucionalidad psicoanalítica incipiente, para llevar una visión psicológica a las barricadas o a la experimentación social o psicosexual. El caso de Wilhelm Reich es, por supuesto, el más notorio, pero contamos con una larga, complicada, interesante y a veces muy fructífera historia de encuentro entre psicoanálisis y marxismo (Dahmer, 1983), de indudable repercusión en varios países de nuestra América.

Reich, recordaremos, merece seguir siendo evocado, cuando se preguntaba, por ejemplo, no por qué un obrero iba a la huelga, sino por qué no iba a la huelga, o cuando anotaba que no se podía entender al fascismo exclusivamente con un análisis racional, o que había que vincularlo con la estructura familiar autoritaria (Reich, 1980).

Podríamos citar, también, entre otros, el ejemplo de Marie Langer, expulsada de Austria por los nazis en los años treinta, y perseguida por la dictadura militar argentina en los años setenta, teniendo que exiliarse en México.

En diferentes momentos la tensión ha atravesado otras perspecti- vas, o se ha desplazado hacia el campo de la ética en la disciplina (Prillieltensky, 1994), y, en lo que se ha conocido como la crisis en la psicología social de los años 60 y 70, que tuvo tanta repercusión en Europa primero, luego en EE.UU. y América Latina, desplazó la atención hacia el campo de la ideología y el poder, y a un cuestionamiento serio de la artificialidad de la producción de conocimiento en el campo y al individualismo metodológico (Martin-Baró, 1983).

Esto eventualmente llevó, por diversos caminos, a la mano de las sacudidas y los cuestionamientos forzados por diversos movimientos sociales (gays/lesbianos, feministas, afros, etc.) al desarrollo de perspectivas criticas, incluyendo las socioconstruccionistas (Gergen, 1996, Ibáñez, 2001) basadas en un pragmatismo a lo Rorty y a un cuestionamiento a la concepción del conocimiento como representación de la realidad y la fundamentación última del saber en un metodologismo y empirismo funcional para la afirmación de poderes opresores.

La premisa mencionada en su ponencia por Angarita y sus compañeros (2008) de un pensamiento neoliberal que "declara inútil la búsqueda de universales en el conocimiento y que se conforma con la afirmación de explicaciones desintegradas que se afirman y se legitiman en el bagaje flotante de los múltiples saberes" parece relevante aquí, con esa paradoja de un pensamiento único, neoliberal, que 
supuestamente se asienta sobre la tolerancia y la diversidad. Pese a las intenciones críticas de los socioconstruccionistas, autodenominados muchas veces como posmodernos, habría que recordar la frase de la investigadora de las memorias sociales brasileña Eclea Bossi $(2002,124)$ :

"Es que después de decir que no hay verdad objetiva, se acaba aceptando que el poder engendra la verdad".

Pese a su indudable influencia, y la importancia de su aporte al pensamiento crítico, al no fundamentarse en una ética de la vida (Ética de la Liberación) y al establecerse sobre la base de un relativismo epistemológico, la fuerte influencia critica de la perspectiva socio construccionista se ha ido desgastando, y lo que parece haber contribuido a producir parece ser una especie de backlash conservador, que ha implicado un fortalecimiento de los intentos de encontrar explicaciones sociobiológicas a la realidad social humana. Esta perspectiva crítica, bien afianzada en Europa y en algunos espacios académicos de Los Estados Unidos, tuvo, a decir verdad, grandes dificultades para insertarse en la región latinoamericana, con la probable excepción de Chile. La realidad ha sido demasiada pesada para ser ignorada.

En el camino han quedado, también, algunos intentos de desarrollar una psicología social mediante la aplicación directa al campo psicológico del materialismo histórico, que se resolvían la mayoría de las veces con planteamientos hacían poco para dilucidar lo psicológico o lo psicosocial, y que e hacen flacos favores a la dialéctica. Una excepción, por supuesto, de enorme originalidad y consistencia, es la de Vygotsky y sus seguidores en la psicología soviética y, en general, en la perspectiva sociocultural.

Más allá del campo científico y del saber, los estancamientos y o las derrotas de diversos movimientos sociales y políticos han afectado a las posiciones más contestarías y críticas en la disciplina, y han llevado, sin duda, a cierto acomodo de intelectuales y profesionales, otrora radicales, domesticando las aristas mas espinosas de la crítica social o transformadora.

Esto se ha evidenciado hasta en la apropiación en los esfuerzos editoriales de la obra y figura de Ignacio Martín Baró, a quien se le disminuye relegándolo a un espacio y temporalidad especifica, restringiendo así su alcance universal, y al que a toda costa se le quiere quitar lo que tenía de marxista (De la Corte, 2001. Ver la discusión de Lacerda, 2006).

Su aporte es, sin duda, uno de los puntos de apoyo más importantes de un impulso crítico y renovador en la psicología latinoamericana, pujando para no caer de nuevo en dependencias intelectuales de los centros del conocimiento 
hegemónicos, y es la base de lo que quiero comentar a continuación. Demuestra la vitalidad que cobra, hoy en día, la realización reciente en San Cristóbal de las Casas, en la Universidad de La Tierra de Chapas, del IX Congreso Internacional de Psicología Social de la Liberación, con más de 1200 participantes, encuentro diseñado y puesto en práctica por las redes de tejido solidario existentes, con una lógica propositiva y de construcción conjunta procurando, como decía su lema "respuestas colectivas a los desafíos actuales de la liberación" (ver www.liber-accion.org).

En todo caso no puede ser mi propósito aquí pretender escribir la historia, larga ya, de los intentos de trabajar desde la psicología atendiendo las complejidades de la vida humana y apuntando hacia la crítica y a transformación social (estos momentos los encontramos, claro está, en el mismo Freud, en algunas partes de su obra). Lo que quiero es compartir y enfatizar un impulso crítico reciente en la psicología y la psicología social, vinculada con la Teología de la Liberación y con una lectura no ortodoxa del marxismo, y, también, con las aspiraciones, las andanzas y los destinos de los movimientos sociales y populares.

Ya lo anuncié. Me refiero a la perspectiva introducida, en su vida truncada a los 47 años por las fuerzas militares salvadoreñas, por Ignacio Martín-Baró, empuje que se ha venido retomando en los últimos años, en un esfuerzo que no ha de- jado de ser contradictorio y problemático, encontrando fuertes escollos en la despolitización y en cierto academicismo, y que en mucho podrá coincidir, en su desarrollo, con el esfuerzo que hemos venido haciendo de trabajar la necesaria renovación del pensamiento crítico.

Lo que está en juego es nada menos que la definición de los sujetos sociales que pueden ser sustento de este intento crítico en esta nueva etapa histórica y las relaciones a definir con estos, los retos epistemológicos y teóricos del mismo y su necesaria articulación con esfuerzos relacionados en otras áreas del saber, en necesaria brega interdisciplinaria.

Podríamos decir, como lo hemos hecho en otros lugares, que se trata de apostar al Principio Liberación, siendo una manera, en nuestro ámbito de acción, de afirmar una ética de vida, una ética de la liberación que implica, como principio fundamental, como se fue definiendo en discusiones sostenidas en años recientes en el Departamento Ecuménico de Investigaciones (DEI) contribuir a producir y reproducir la vida humana, en su corporeidad, en lo simbólico y pulsional, en sus características específicas y diversas, y en comunidad.

En su trayectoria intelectual truncada por las balas asesinas del ejercito y el gobierno salvadoreño, un Martín-Baró crecientemente frustrado con la incapacidad de la psicología para dar respuestas a las 
necesidades de las mayorías populares en el continente, y de salirse de su esfera autocontenida, pasó de efectuar una crítica profunda y una revisión de la psicología social dominante en su tiempo, a esbozar el horizonte de una posible "Psicología de la Liberación", sugiriendo desafíos y tareas que siguen cobrando vigencia, a juicio de muchos de nosotros para una reconfiguración de la perspectiva psicológica, de manera crítica, en función de la transformación social y el bienestar de las mayorías.

Los debates surgidos desde entonces clarifican, a mi juicio, la necesidad de que esta búsqueda se vincule con otras visiones disciplinarias, y se ubique en un marco ético y filosófico mayor, así como la necesidad de contribuir a crear, como ha señalado Duchrow, (2006) nuevos "grandes relatos" (un "nuevo gran relato con historias esperanzadoras" lo llama) que permitan, sin mecanicismos o ilusiones de perfección, apuntar hacia nuevas utopías. Es particularmente urgente el diálogo entre psicología de la liberación y economía crítica, como empezó a esbozarse en nuestro medio en el II Encuentro Nacional de Psicología de la Liberación realizado en julio de este año en la Universidad Nacional.

En una conferencia, ya famosa, dictada en la Universidad de Puerto Rico en el año de 1986, MartínBaró proponía tres grandes desafíos para una psicología liberadora. Estructuraré el resto de mi discusión en torno a estas ideas centrales.
El primero implicaba la necesidad de las psicología de descentrarse de sí misma, lo que llamaba "la liberación de la psicología", es decir, dejar de prestarle tanta importancia a una parcelación de la realidad que convertía a la disciplina en "dueña" de algunas de sus partes, o a un pretendido cientificismo y búsqueda de status y prestigio, para basar su quehacer, mas bien, en el trabajo en torno a las problemáticas de las grande mayorías de nuestros pueblos: a trabajar el conflicto, donde antes solo se veía la adaptación, a trabajar el desempleo, donde antes solo se veía la selección de personal, a trabajar los devastadores efectos de la guerra y la represión, donde antes se presumía la estabilidad y la homogeneidad, a trabajar la exclusión, donde antes se asumía una aparente igualdad, a trabajar las heridas sociales y colectivas (trauma psicosocial), donde antes solo se veía el trauma individual, a volver a retomar el extraviado (en la psicología) tema de las necesidades, cuando solo se avizora el consumo.

Este énfasis en la actuación sobre la realidad para transformarla entra de inmediato en tensión con los socioconstruccionistas, que aconsejan o dictaminan no hablar de realidades, y tiene mucho que ver con el "ver, juzgar y actuar" al que apela Angarita (2008). En todo caso lleva, en un esfuerzo que, como veremos, es necesariamente colectivo, a discernir cuales serian problemáticas prioritarias para ser trabajadas, teorizadas, y para 
ser abordadas mediante el trabajo priorizado por la disciplina, es decir, el trabajo con la gente. Hemos propuesto, en otro lugar, siguiendo en buena medida discusiones en que hemos participado con Franz Hinkelammert y otros compañeros, cuatro ámbitos urgentes:

1. El de la lógica de una seguridad nacional globalizada (Hinkelammert, 2005): que lleva no solo al nuevo lugar ocupado por el discurso y la práctica de la tortura en los dominios de la disciplina (Dobles, 2006), sino, también, a la criminalización de los movimientos sociales, la ubicación de la migración, en la globalización con conducción neoliberal como, un asunto de seguridad nacional, y a la relativización y redefinición del trabajo relacionado con los derechos humanos, tan importante para la psicología latinoamericana en las últimas dos décadas.

2. Las implicaciones y los efectos del fundamentalismo de mercado, que impregna acrítica y directamente las más diversas expresiones de la práctica psicológica: educativa, laboral, clínica. Esto implica también desmontar los presupuestos psicológicos del discurso neoliberal y trabajar una concepción de la subjetividad y la intersubjetividad en que se rescaten los valores de la solidaridad, de la inclusión, de la perspectiva de mundos en que "quepamos todos". Retomar y trabajar la "ética del bien común" que ha puesto en discusión Hinkelammert, se convierte en tarea crucial. Implica, también enfrentar al individualismo, afirmando la construcción de esfuerzos colectivos.

3. El impulso a mecanismos de participación democrática reales, a que en la microfísica y también en la microfísica del poder las personas y los pueblos tengan mayor autonomía, libertad real, capacidad de decisión y de control sobre sus vidas. Tenemos aquí, en la psicología social y comunitaria latinoamericana, un enorme y valioso caudal de experiencias a rescatar, bajo la influencia de Fals Borda, Freire y otros autores (Dobles, 2008), desde la investigación participativa, aunque no podemos mistificarla, ya que la experiencia demuestra que puede utilizarse hasta para fines contrainsurgentes. No obstante, tenemos aquí un ámbito de acción fundamental, que implica desde procesos electorales nacionales que terminan siendo decididos por grupos minúsculos de personas en puestos claves, o la posibilidad real de participación, efectiva, en un grupo o una comunidad determinada.

4. Por último, ante un capitalismo que no puede sino ser rapaz, y un orden social y económico mundial que tiene, aunque sea 
como efecto no intencional, la tendencia a destruir el mundo en que vivimos, se coloca ante nosotros, con mayor urgencia, el reto de trabajar la relación ser humano/naturaleza, la sustentabilidad, la protección de los recursos naturales. Esto, obviamente, no se queda solo en lo natural, porque inevitablemente pasa por los arreglos existentes en el orden social. En una valiosísima experiencia desarrollada el pasado mes de octubre por la Red Costarricense de Psicología de la Liberación, llevamos a cabo un intenso seminario sobre psicología de la liberación y medio ambiente con la participación de activistas ecologistas, y de este encuentro emergieron una serie de tareas urgentes posibles para trabajar en este terreno (Dobles, 2008). Entre otras cosas, se perfiló el reto, en nuestro contexto costarricense, de pensar y conceptualizar el miedo, sus expresiones y consecuencias, y de articular, ahora desde el Colectivo formado en octubre, un proyecto de acompañamiento a activistas y movimientos sociales.

En todo caso, la liberación de la psicología a la que aludía MartínBaró implica, sobre todo, poner en un primer plano el abordaje, teórico y metodológico, práxico, de los problemas sociales prioritarios que amenazan la existencia humana y las posibilidades de una vida digna.
El segundo gran desafío planteado por Martín-Baró es epistemológico. Incita a que la psicología supere el positivismo, entendido como afirmación del dato empírico, constatable, en detrimento de la realidad posible, en circunstancias diferentes, retando también el hedonismo presente en las teorías psicológicas, que hace imposible, por ejemplo, considerar el tema de la solidaridad o, siquiera, el bien común como perspectiva, y que reta a trascender visiones homeostáticas, no conflictivas, del orden social, y, podríamos agregar, personal. Este último aspecto es particularmente relevante, cuando el orden social y económica implica la imposibilidad de una vida y de un futuro para todos.

Un pensamiento crítico en psicología tendría que partir de la precariedad del orden social insistente, y de la necesidad de su transformación a través de la acción humana.

Lo que notamos, hoy en día, mas bien, en las instituciones en que se hace psicología, es una presión creciente hacia una especie de tecnocracia del saber, con fuertes rasgos positivistas, y no ha sido poco común ver la transformación de quienes en algún momento sustentaban posiciones más críticas hacia una especie de acomodamiento cientificista, lo que de paso, trae de vuelta, con fuerza, uno de los temas enfatizados por Martín-Baró o en su obra: la pretendida neutralidad de la psicología y del psicólogo. Dicho en otros términos la expulsión del 
campo del saber y de la práctica de los valores sustantivos en pro de los valores instrumentales. Por todo lado, la eficacia parece ser la palabra del día.

El último desafío para una psicología de la Liberación, desde la propuesta martinbaroniana, y muy en sintonía con el énfasis puesto en la ortopraxis por la teología de la liberación, implica la necesidad de que la psicología emprenda una nueva praxis, trabajando desde la perspectiva de las víctimas, y de la necesaria transformación de sistemas opresores.

Esto opera en doble vía, porque lleva no solo a la necesidad de articular el desarrollo de una saber y de una práctica con el quehacer de aquellos sectores sociales y populares que luchan por una vida más justa para todos, sino que implica, también, reconocer que en ese propósito, la acción colectiva de quienes se comprometen con su desarrollo implica, a la manera de la constitución de una comunidad crítica en la forma que lo entiende Dussell (1998), reconocer la necesidad de transformarse, también, en sí mismos, en movimiento social. Es decir, buscar las modalidades organizativas y colectivas que sin reproducir esquemas verticales y autoritarios propicien la y sostengan la constitución de esta nueva praxis y de este nuevo saber. Eso es, de alguna manera, lo que hemos intentado hacer a nivel regional, articulando un tejido comprometido que, superando una serie de obstáculos academicistas y despolitizadores, acaba de expresarse en el Noveno Congreso Internacional de Psicología Social de la Liberación, ya comentado, y lo que hemos venido haciendo en Costa Rica con el funcionamiento de la Red Costarricense de Psicología de la Liberación, y, ahora, con la Asociación Colectivo Costarricense de Psicología de la Liberación.

Esto roza con un tema que fuera planteado en una de las primeras reuniones del grupo de Pensamiento Crítico, a que nos podemos referir, abusando de la retórica "militante", como el asunto del "relevo de cuadros". Hemos visto como, en algunos ámbitos académicos en que actúan e incluso ocupan puestos de importancia personas que vienen del compromiso y la participación política (el 68, por ejemplo) se reproducen, en laberintos institucionales verticalistas, métodos y prácticas que reducen la posibilidad de participación, desarrollo o incluso abordaje de problemáticas recientes, lo que obliga a estos compañeros y compañeras a enfrentarse a la dura realidad de que a la hora de la verdad los auditorios están vacíos, y los más jóvenes están en otra cosa.

Parece esbozada, entonces, una estrategia para la reconstrucción de un pensamiento critico y una praxis comprometida de la psicología, que no repita errores del pasado, y que se articule interdisciplinariamente y en relación directa con las fuerzas sociales que pueden sustentar y sostener la transformaciones necesarias en el orden capitalista neoliberal. 
Esta estrategia pasa, entonces, por el desarrollo de un esfuerzo colectivo, con sus concomitantes organizativos, que ponga en un primer plano problemáticas cruciales para nuestros pueblos, con la capacidad de establecer interlocuciones y diálogos con otros campos: como la economía, las ciencias políticas, la teología, y que ponga en primer plano los discernimientos necesarios acerca de las subjetividades necesarias para sostener mundos en que quepamos todos, con respeto a las dimensiones comunitarias, a las diferencias, y al medio ambiente.

Es clara la lección, no obstante, de que, también en este campo, será difícil avanzar en un esfuerzo crítico sin esbozar de manera mas clara un proyecto de sociedad, de mundo, en que no impere el fundamentalismo de mercado.

\section{Bibliografía}

Angarita, C. (2008) Elementos epistemológicos para el análisis teológico de la realidad social. Documento presentado al Encuentro Internacional del Grupo de Pensamiento Crítico, Heredia.

Bosi, E. (2003) O tempo vivo da Memoria. Ensaios de Psicología Social. São Paulo: Atelie Editores.

Chodorow, N. (2003) El poder de los sentimientos. La significa-
Los retos son muchos. Una Psicología de la Liberación, concebida de esta manera, tendría que actuar y teorizar acerca de muchos asuntos, en el campo de lo cotidiano, de los comunitario, de lo intersubjetivo, y no podrá obviar, tampoco, como bien lo explica Chodorow (2003) el ir mas allá de las determinaciones externas de las acciones humanas para, como esbozásemos en la definición del "principio liberación" con que empezamos este escrito, trabajar los significados emocionales, lo pulsional, que matiza los acontecimientos humanos. Esto hace necesario, a mi juicio, el encuentro de lo que ya podemos llamar una cierta tradición de "Psicología de la Liberación" con aportes del psicoanálisis, asumido también críticamente. Este no es un asunto sencillo, pero es necesario, y es, en buena medida, asunto pendiente.

ción personal en el psicoanálisis, el género y la cultura. Buenos Aires: Paidos.

Dahmer, H. (1983) Libido y Sociedad. Estudios sobre Freud y a Izquierda freudiana. México: Siglo XXI.

De la Corte, L. (2001) Memoria de un compromiso. La Psicología Social de Ignacio Martin-Baró. Bilbao: Editorial Descleé de Brouwer, S.A.

Dobles, I. (2008) Psicología de la Liberación y Medio ambiente. 
www.liber-accion.org. Colocado el 5 de octubre.

Dobles, I. (2006) Psicología de la liberación y procesos sociales y políticos en América Latina: desafíos y posibilidades. www. rebelion.org , 8 diciembre.

Dobles, I. Memorias del dolor y experiencias de las Comisiones de la Verdad en América Latina. San José: Editorial Arlekin, (en prensa).

Dobles, I. 2005. "Wilhelm Reich y el fascismo" en Actualidades en Psicología, vol 19, 106, 96111.

Duchrow, U. (2006) "Llegar a ser humano solidariamente en contra de la destrucción neoliberal: liberalización de traumatizarían, codicia, miedo y estrés en perspectiva teológica" en Dobles, I., Baltodano, S., Leandro, V. Psicología de la Liberación en el contexto de la globalización neoliberal. Acciones, reflexiones, y desafíos. San José: Editorial de la Universidad de Costa Rica.

Dussell, E. (1998) Ética de la Liberación en la época de la globalización y la exclusión.

Gergen, K. (1996) Realidades y relaciones. Aproximaciones a la construcción social. Buenos Aires: Paidos.

González, M. (2008) Psicología del neoliberalismo. www.liberaccion.org. Colocado el 14 de enero.
Hinkelammert, F. (2005) La transformación del estado de derecho bajo el impacto de la estrategia de Globalización. San José: Material de discusión. Departamento Ecuménico de Investigaciones.

Ibáñez, T. (2001) Psicología social construccionista, Guadalajara: Universidad de Guadalajara.

Ibáñez, T. (2001) Municiones para disidentes. Realidad-verdadpolítica. Barcelona: GEDISA Editorial.

Lacerda, F. (2006) " ¿Liberarse de qué? ¿Liberarse para qué? Notas sobre marxismo, anticapitalismo y psicología de la liberación" en Dobles, I., Baltodano, S., Leandro, V. Psicología de la Liberacion en el contexto de la globalización neoliberal. Acciones, reflexiones, y desafíos. San José: Editorial de la Universidad de Costa Rica. 201 a 208.

Leandro, V., Montoya, J. (2006) "Los discursos psicológicos en los medios de comunicación costarricenses; apuntes para una reflexionen Dobles, I., Baltodano, S., Leandro, V. Psicología de la liberación en el contexto de la globalización neoliberal. Acciones, reflexiones, y desafíos. San José: Editorial de la Universidad de Costa Rica, 383 a 398.

Martín-Baró, I. (1986) "Hacia una Psicología de la Liberación" Boletín de Psicología. UCA, 22, 219-231. 
Martín-Baró, I. (1983) Acción e Ideología. Psicología Social desde Centroamérica. San Salvador: UCA Editores.

Prilleltensky, I. (1994). The morals and politics of psychology: psychological discourse and the status quo. State University of New York Press.

Reich, W. (1980). Psicología de masas del fascismo. Madrid: Editorial Bruguera.
Rose. N. (1996). The Invention of the Self. Cambridge University Press.

Sampson, E. (1996). "Celebrando al otro: una interpretación dialógica de la naturaleza humana" en Cordero, T., Dobles, I, Pérez, R. Dominación Social y subjetividad. Contribuciones de a Psicología Social. San José: Editorial de la Universidad de Costa Rica. 